\title{
1.1 Beam-beam Issues in eRHIC
}

\author{
Y. Hao and V. Ptitsyn \\ Mail to: yhao@bnl.gov, vadimp@,bnl.gov \\ Brookhaven National Laboratory, Upton, NY 11973, USA
}

\subsubsection{Introduction}

eRHIC, a future electron-ion collider developed at BNL, aims to provide electron-ion collisions by adding a new electron accelerator to the existing RHIC ion accelerator rings [1]. To assure that eRHIC will become an invaluable tool, we must ensure that

the energy of electron beam and nuclei is adjustable over a large range (5-30 GeV for electrons, $50-250 \mathrm{GeV}$ for protons)

$>$ high luminosity (exceeding $10^{33} \mathrm{~cm}^{-2} \mathrm{~s}^{-1}$ )

$>$ high polarization for both the electron and proton beams

Possible options for accelerating high average current electron beam include an energy-recovery linac (ERL) or a storage ring. Since the proton beam is circulating in the accelerator ring, corresponding collision schemes are called as the linac-ring and the ring-ring. The electron accelerator option based on the electron storage ring was studied on earlier stages of eRHIC design [2]. It was found that the luminosity in this ring-ring scheme was limited by the beam-beam interaction to the level of several units of $10^{32} \mathrm{~cm}^{-2} \mathrm{~s}^{-1}$. Thus, the eRHIC design, using an ERL to accelerate the electron beam, became a preferred one and has been developed in recent years. In ERL-based eRHIC the luminosity can exceed the value of the ring-ring scheme by at least an order-of-magnitude because the electron beam is used only once, and therefore, the opposing proton beam can disrupt it more. The high average current of polarized electrons has to be provided by a polarized electron source, which presents an important R\&D item for the ERL-based eRHIC design. The beam-beam effects in the linac-ring scheme have several specific features and challenges. Since there has been no a collider operating with the linac-ring collisions, the thorough exploration of the features of the beam-beam interaction in ERL-based eRHIC is required both analytically and by the simulations. Several studies of the beam-beam effects in the linac-ring scheme were done in early nineties, when this scheme was considered at the design of B-factories [3].

During the collision the electron beam undergoes a large beam-beam phase advance that entails a considerable mismatch between the beam distribution and the design electron optics. Furthermore, the phase advance exhibits a dependence on the betatron amplitude because of the nonlinearity of the beam-beam force, so causing the distortion of the distribution in the transverse phase space, the so-called 'disruption effect'. Both the mismatch and the disruption effects must be studied carefully to ensure the proper transportation of the electrons after the collision in the decelerating stage, which is required by the energy-recovery process. Such studies will determine the required apertures of the linacs and the transport paths. 
Another key feature of the linac-ring scheme is that a head-tail type of instability may develop in proton beam, named the "kink instability". The electron beam deforms during its interaction and transfers information on the head of the proton beam to the tail. Hence, the proton beam experiences a wake field created by its interaction with the electron beam. We must ensure the suppression of this instability if its parameters are beyond the instability threshold.

In addition to the possible coherent instability of the proton beam, it might be degraded by the nonlinearity of the beam-beam force. Nonlinear diffusion is enhanced because the electron beam is focused by the beam-beam interaction to a smaller rms beam size, resulting in considerable enlargement of the beam-beam force acting on the proton beam. This effect, usually referred as the "pinch effect", must be mitigated to reduce the growth rate of the proton beam transverse emittance.

Table 1. Beam parameters of ERL-based eRHIC

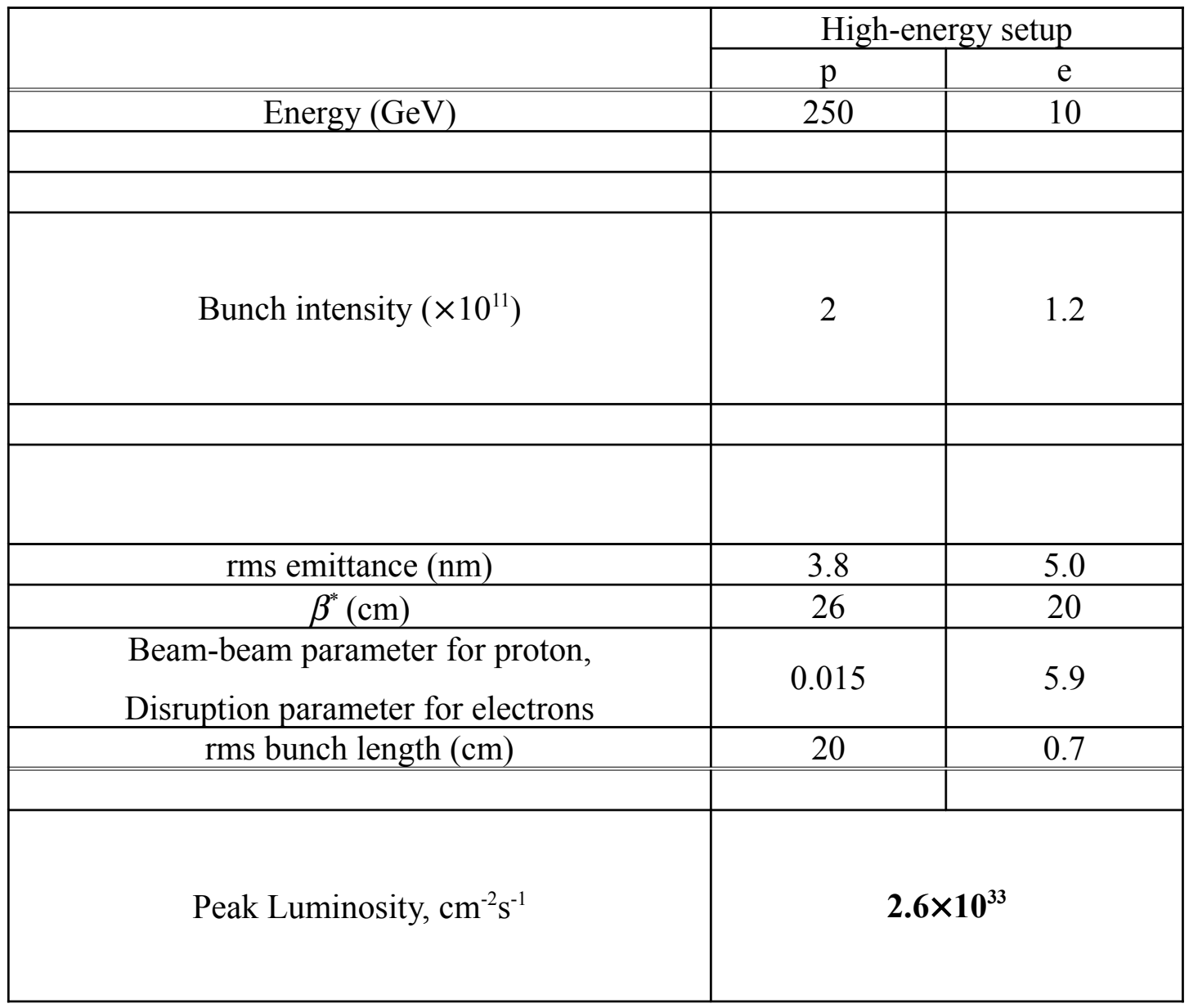

Another important challenge is related with the random errors in the initial condition of the electron beam. The position jitter of the electron beam becomes a random dipole field error for the proton beam, while the fluctuations of the intensity and beam size of the electron beam create quadrupole errors. While these deviations are inevitable, they can be lowered by improving the stability of the electron source and the electron beam transport. 
The effect of the beam-beam interactions on the proton beam is characterized by the beam-beam parameter. The beam-beam parameter limit of 0.015 was accepted for the protons on eRHIC, following the experience with proton-proton collisions in RHIC. For an electron beam, the disruption parameter $\left(d=\sigma_{i} / f\right)$ presents a better measure of the beam-beam effects, where $\sigma_{z}$ is the proton beam bunch length and $f$ is the focal length of the beam-beam lens. The disruption parameter indicates the number of betatron oscillations the electrons perform inside the proton beam [4].

\subsubsection{Electron Beam Disruption Effect}

In eRHIC, the effect of beam-beam interaction on the electron beam is much larger than that on the proton beam. Table 1The beam-beam force disrupts the electron beam distribution considerably after just one collision with protons; meanwhile, the distribution of the proton beam changes very slowly. To investigate the evolution of the electron beam in one collision process, we can assume that proton beam is rigid. Then, we can distinguish two components in the disruption of the electron beam. First, the nonlinearity of the beam-beam force distorts its distribution, since the phase advance differs at various transverse amplitudes, and increases its transverse emittance. Second, the linear part of the beam-beam interaction (strong focusing) engenders a mismatch between the electron distribution in phase space and the aperture's shape, as defined by the design lattice without collisions. The deformation of the electron beam distribution by the beam-beam interactions must be minimized in order to guarantee successful transport of the electron beam at the deceleration stage of the energy recovery process. Because of that the detailed studies of the electron beam disruption in eRHIC have been done [5].

\subsubsection{Linear Approximation}

We start our discussion from the linear approximation in which we treat the beam-beam force as a thick focusing element in both transverse directions, with arbitrary longitudinal profile that is determined by the proton beam longitudinal distribution. We discuss here a uniform distribution, and a Gaussian distribution.

The linearized beam-beam force rotates the electron beam in phase space, causing a mismatch between the electron-beam distribution and the acceptance of the electron lattice downstream. Consequently, the emittance of the electron beam increases if we measure it with the optics functions of the lattice; we name this as "effective emittance". We distinguish it from the former emittance calculated from the beam distribution that we call the "geometric emittance".

To mitigate the growth of the effective emittance, we seek a solution for the optics of the electron beam so that, after the beam-beam interaction, its distribution exactly matches the lattice downstream. With this solution, at the position far away from the interaction point one cannot conclude whether there was a beam-beam interaction at all. In the absence of the beam-beam interaction, the interaction region 
lattice can be characterized by the optics parameters $\left(\beta^{*}\right.$ and $\left.s^{*}\right)$, which represents the waist beta-function and its position. If the timing of the collision is perfect, the beambeam force exercised by the electron beam is symmetric to the IP. Therefore, the perfect matching solution exists only if the lattice design also is symmetric $\left(s^{*}=0\right)$. Thus, the only variable is the beta function at IP.

In figure 1, we illustrate the evolution of optics functions in the IR at different design $\beta^{*}$. The proton beam has a Gaussian longitudinal distribution, with rms bunch length $0.2 \mathrm{~m}$. Table 1 lists the other relevant proton beam parameters. The electron beam propagates from the right side of the figure to the left. In general, the optics with beam-beam interaction deviates from those without it. At $\beta^{*}=1 \mathrm{~m}$, the beta function after collision is larger with beam-beam effect, while it is smaller when $\beta^{*}=0.2 \mathrm{~m}$. We can calculate the exact matching $\beta^{*}$ with a numerical solver. The result is $\beta^{*}=0.225 \mathrm{~m}$. In figure 2 , we show that the optics functions match each other at both the entrance and exit of the collision region; deviation is limited to the region where the proton beam is present.
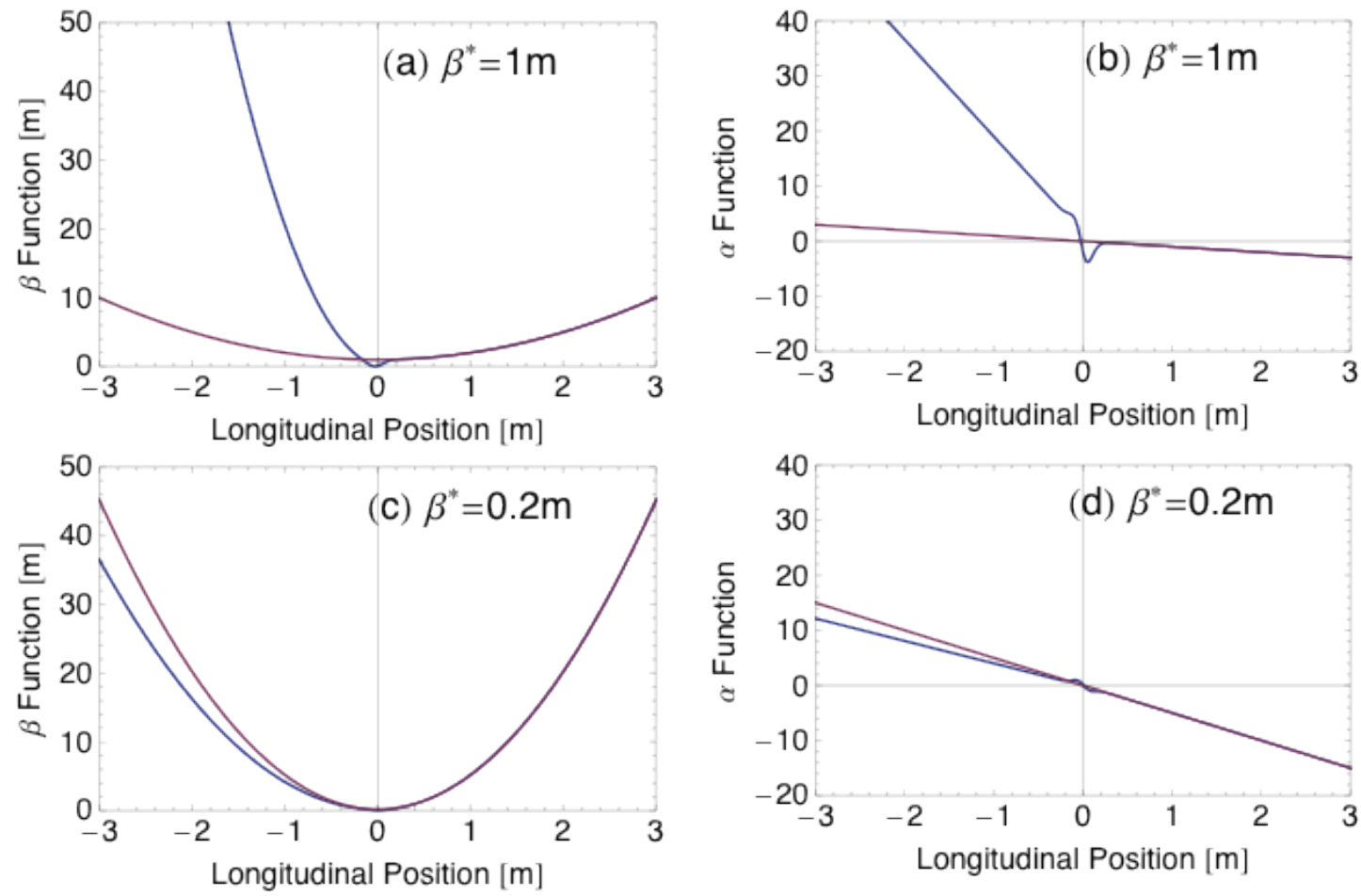

Figure 1. Comparison of the beta function at IR at different beta functions. The red lines are cases without beam-beam interaction (design optics); the blue ones are those with beam-beam interaction. 

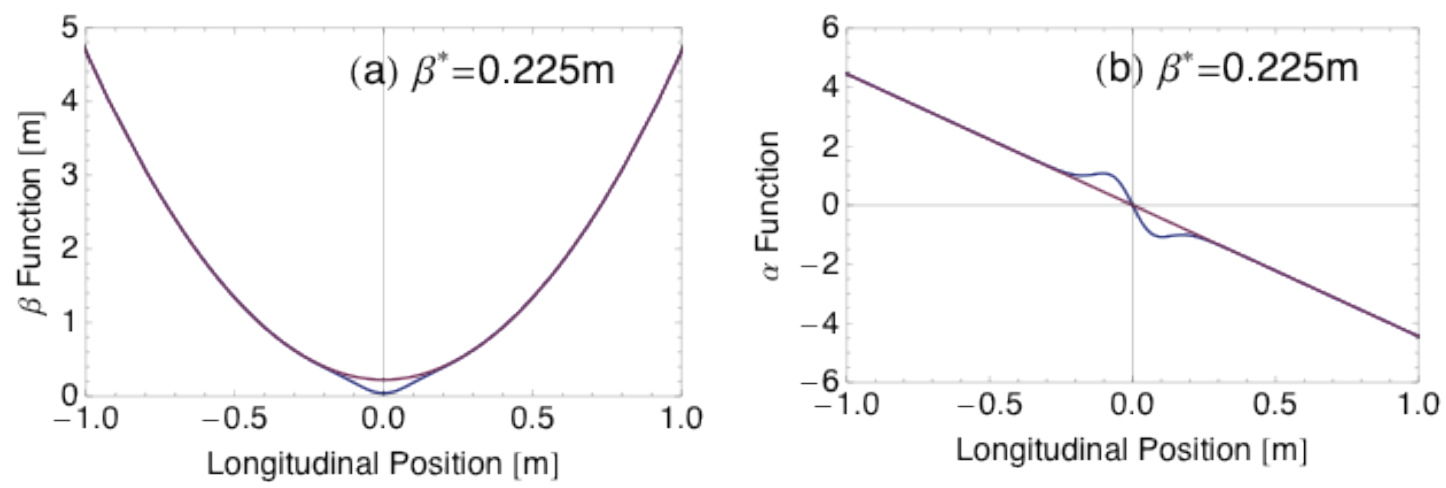

Figure 2. The optics functions of a perfect matching design $(\beta=0.225 \mathrm{~m})$. The red lines are the cases without beam-beam interaction (design optics); the blue ones are those with beam-beam interaction.

The exact matching solution can be derived analytically [5] if we assume that the proton beam has a uniform longitudinal distribution:

$$
\beta^{*}=\frac{\sqrt{1-k^{2} L^{2}+k L[\tan (k L)-\cot (k L)]}}{k}
$$

Here, the proton beam has the uniform distribution [-2L, $2 L]$ and the focusing strength is defined as $k=\left(2 f_{e} L\right)^{-1 / 2}$. This relation shows that the matching condition does not always exist for all parameters, as demonstrated in figure 3 .

When $k L$ falls within the region $[0, \pi / 2]$, the matching solution always exists. Figure 3

Equivalently, this requires that the disruption parameter D is less than 5.7. For a Gaussian longitudinal distribution, we can similarly determine the requirement for the maximum disruption parameter. In this case, the disruption parameter that assures a matched optics solution should be less than 8.3. As figure 3 shows there are also another $k L$ ranges, at higher $k L$ values, which allow the exact matching solution. Figure 3 


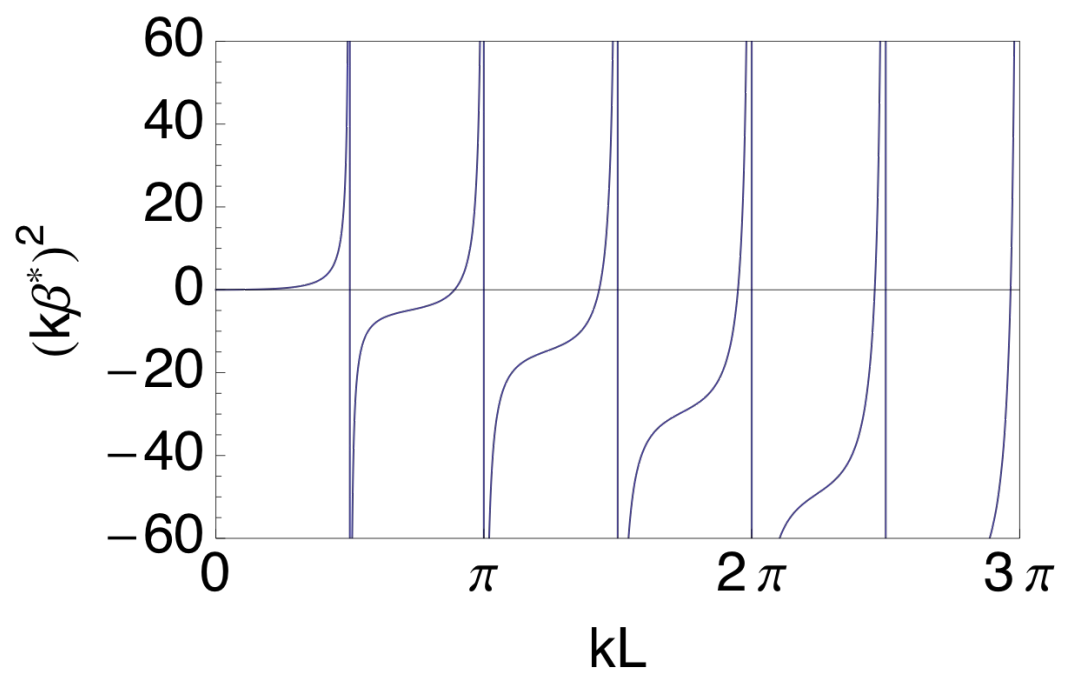

Figure 3. The solution of the design beta-function for the exact matching solution for uniform proton distribution. The real solution exists only when $(k \boldsymbol{\beta})^{2}$ is positive.

\subsubsection{Nonlinear Effects}

If we account for nonlinearity, the electrons with various betatron amplitudes have different phase advances. Accordingly, electron distribution is distorted, especially at large betatron amplitudes, thereby altering the geometric emittance of the electron beam. To explore the resulting deterioration of the beam emittance, we undertook a simulation of its dynamics.

In this simulation, we assumed that the proton beam is rigid (strong beam) and has the parameters listed in Table 1. We ignored the length of the electron beam bunch; therefore, its longitudinal profile is a delta function. The optimized electron beta function from the linear approximation does not necessary fit well in the nonlinear case. Here, for comparison, the cases of two different electron $\beta^{*}(0.2 \mathrm{~m}$ and $1 \mathrm{~m}$ ) are illustrated. The transverse emittance of the electron beam is changed correspondingly to match that of the proton beam.

In figure 4 and 6 , we depict the evolution of the rms size of the electron beam, its geometric emittance and its effective emittance. The electron beam travels from the right side to the left. The evolution of the electron beam depends strongly on the design electron-beam optics $\left(\beta^{*}\right)$. The geometric emittance increases as the electron beam moves through the opposing proton beam. This increment ratio for the low $\beta^{*}$ is well below than that for the high $\beta^{*}$ case. The effective emittance, mainly contributed by the advance in linear phase, exhibits about a 5 times difference between these two examples. The low $\beta^{*}$ case certainly is the desirable one since it has a lower emittance after collision.

Figures 5 and 7 show the phase-space distribution after the collision of the electron beam, along with the rms emittance ellipse. We back-traced those distributions to IP so to compare them with the design optics $\left(\beta^{*}\right)$. The mismatch between the beam distribution and the design optics is smaller for low $\beta^{*}$. 


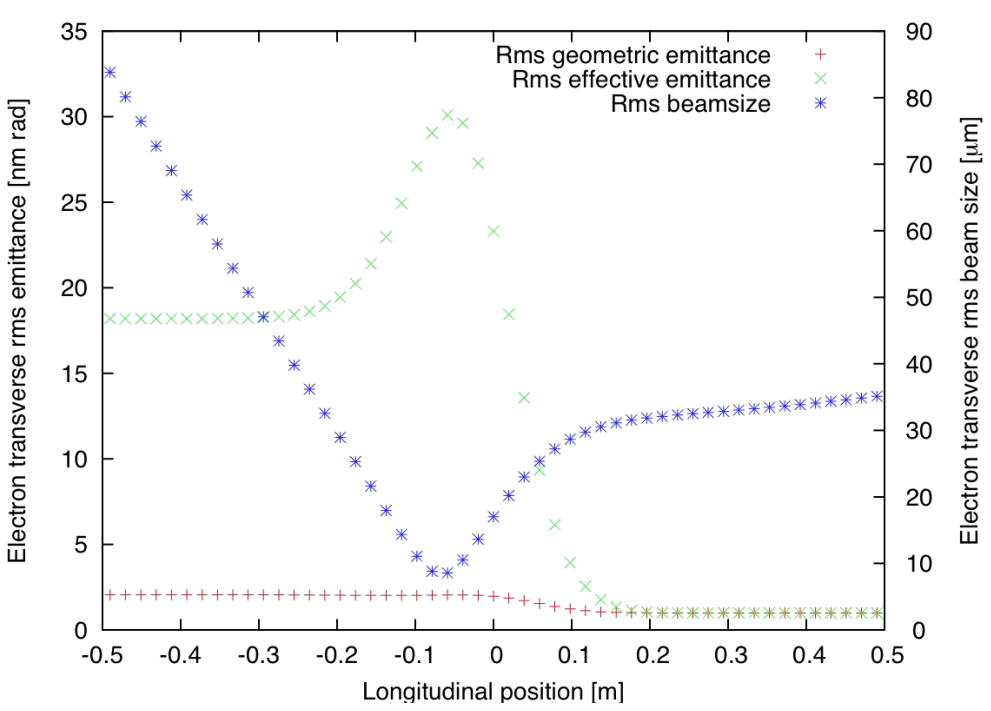

Figure 4. Evolution of beam size and rms emittance (geometric and effective) of the electron beam for $\beta^{*}=1 \mathrm{~m}$

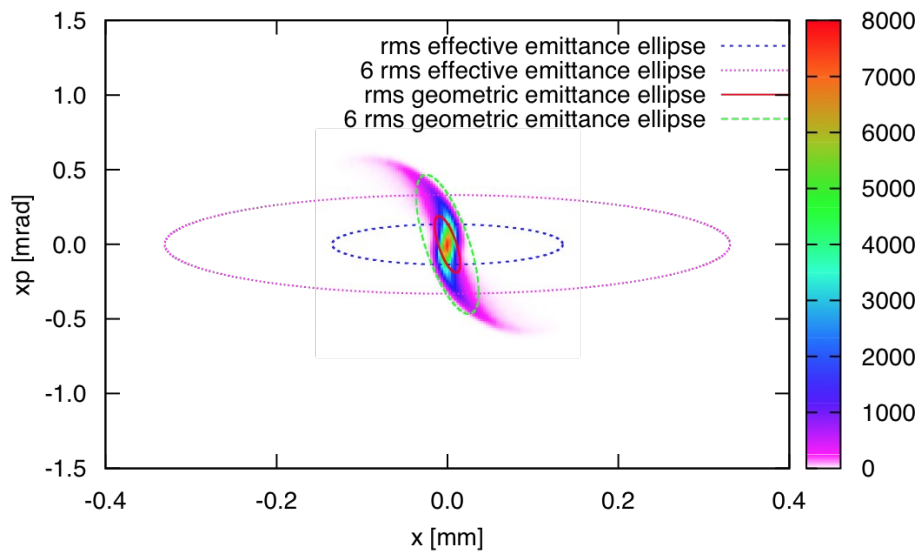

Figure 5. Phase-space distribution of the electron beam after collision for $\beta^{*}=1 \mathrm{~m}$, back-traced to IP. The rms and 6 rms ellipses for both the geometric and the effective emittance are plotted. 


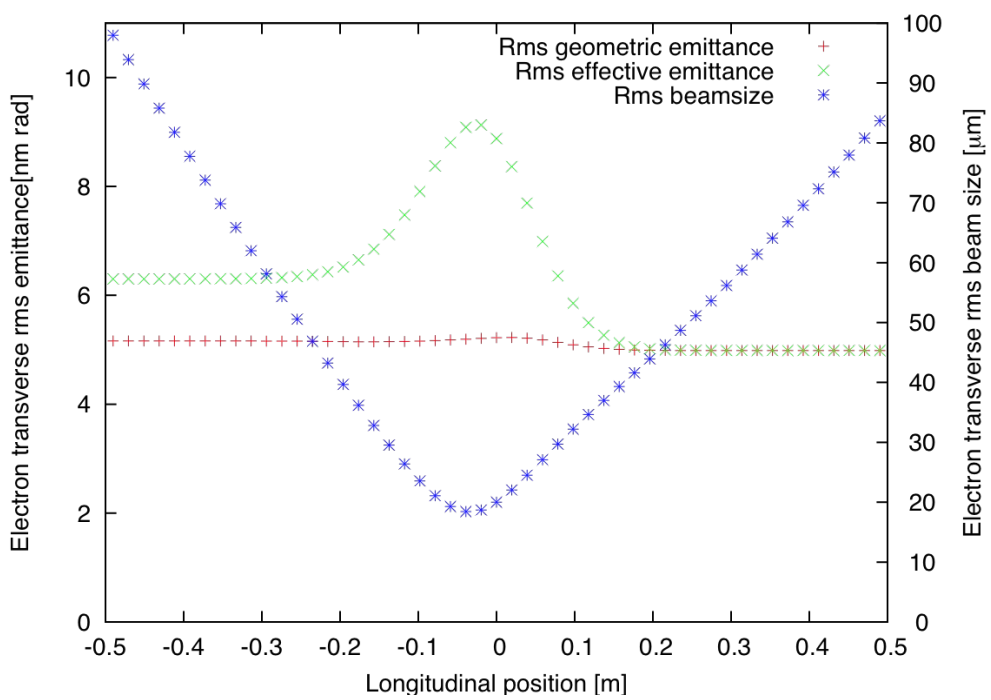

Figure 6. Evolution of the beam size and rms emittance (geometric and effective) of the electron beam for $\beta^{*}=0.2 \mathrm{~m}$.

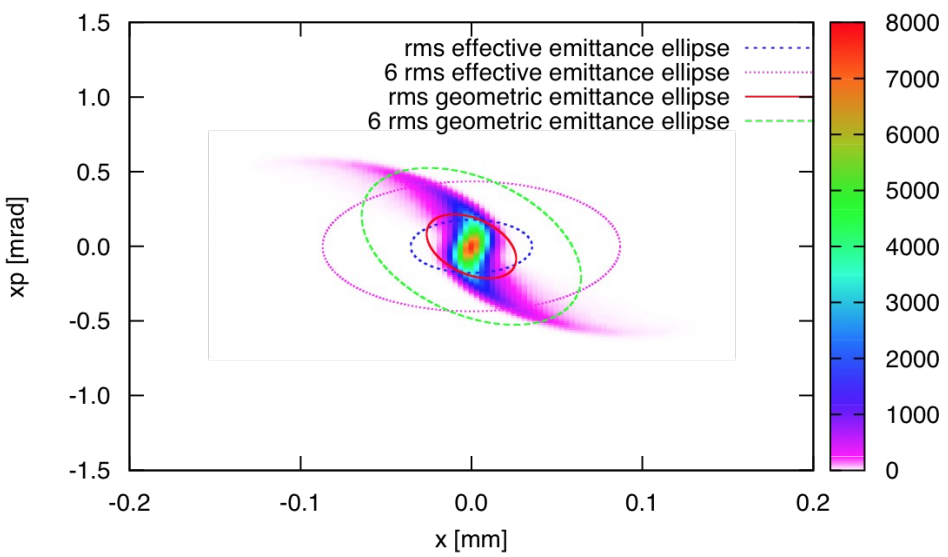

Figure 7. Phase-space distribution of the electron beam after collision for $\beta^{*}=0.2 \mathrm{~m}$, back-traced to IP. The rms and 6 rms ellipses for both the geometric- and effectiveemittance are plotted.

Another notable effect is the so-called "pinch effect" whereby the opposing proton beam greatly focuses the electron beam. In both of these above two cases, the minimum size of the rms electron beam, respectively, reaches 8 microns $\left(\beta^{*}=1 \mathrm{~m}\right)$ and 16 microns $\left(\beta^{*}=0.2 \mathrm{~m}\right)$. The direct consequence is an enhancement in luminosity because the average size of the rms electron beam shrinks during the beam-beam interaction. Table 2 lists the integrated luminosity from different initial electron optics. The pinch effect augments luminosity, while the hourglass effect degrades it. These two effects countermand each other, so that luminosity is higher than its nominal value when $\beta^{*}$ is larger than $0.2 \mathrm{~m}$. 
Table 2. Luminosity for different electron beam parameters

\begin{tabular}{cccc}
\hline \multicolumn{3}{c}{ Initial electron beam parameters } & \multirow{2}{*}{ Luminosity $\left[\mathrm{cm}^{-2} \mathrm{~s}^{-1}\right]$} \\
\hline Emittance $[\mathrm{nm}-\mathrm{rad}]$ & $\beta^{*}[\mathrm{~m}]$ & Waist position $[\mathrm{m}]$ & \\
\hline 1 & 1 & 0 & $3.7 \times 10^{33}$ \\
\hline 4 & 0.25 & 0 & $3.3 \times 10^{33}$ \\
\hline 5 & 0.2 & 0 & $3.0 \times 10^{33}$ \\
\hline 10 & 0.1 & 0 & $2.3 \times 10^{33}$ \\
\hline
\end{tabular}

However, the intensification of luminosity is not without its drawbacks. The beam-beam parameter for the proton beam becomes too large when the electron beam reaches its minimum rms size. For example, for the design $\beta^{*}=1 \mathrm{~m}$, the minimum beam size is 8 microns, one quarter of the nominal waist size without the collision. There, the maximum focusing strength of the beam-beam force has a value 16 times that of the designed value. Furthermore, the shrinkage of the electron beam size changes its distribution. The histogram, shown in Figure 8, demonstrates the modification of the transverse beam distribution by the beam-beam interaction .
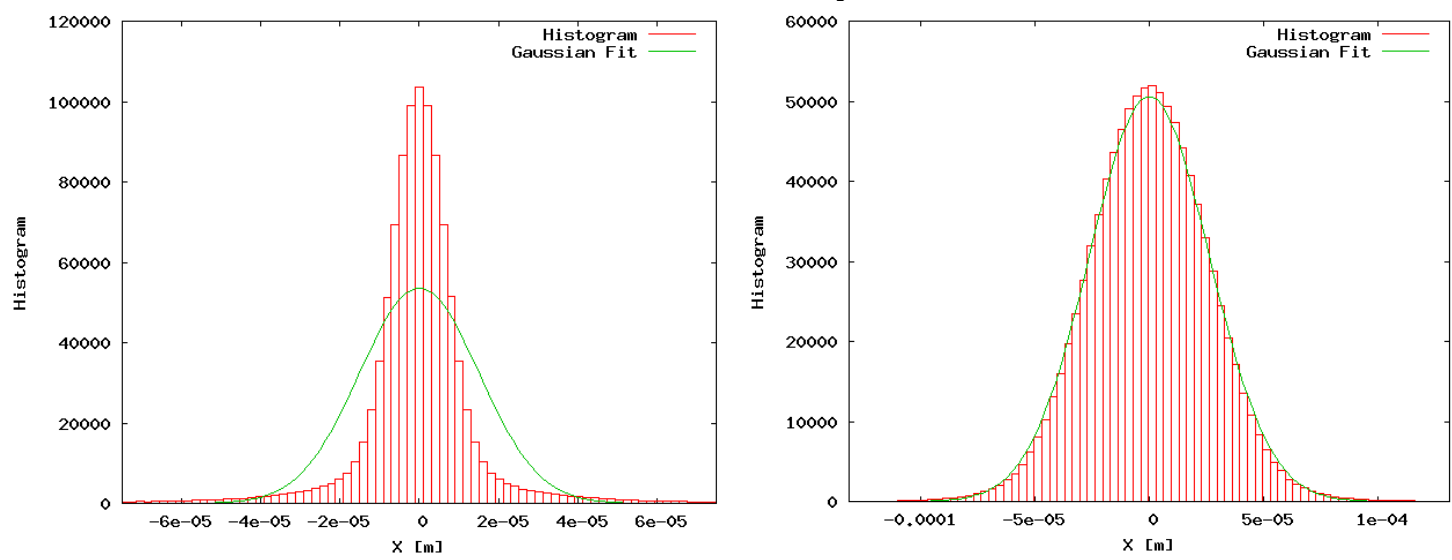

Figure 8. Histogram of the electron transverse positions after beam-beam collision. Left: the initial electron rms emittance is $1 \mathrm{~nm}$-rad and $\beta^{*}$ is $1 \mathrm{~m}$ at IP. Right: the initial electron rms emittance is $5 \mathrm{~nm}$-rad and $\beta^{*}$ is $0.2 \mathrm{~m}$ at IP. The green curve corresponds to the Gaussian-function fit based on the electron beam rms size and histogram data.

Figure 8 reveals that, if the initial beta function is large, the electron distribution forms a denser core and longer tail than a Gaussian distribution with same rms size. However, the deformation is almost negligible when beta function is $0.2 \mathrm{~m}$. The pinch effect not only shrinks the electron beam size but also distorts its distribution so to entail an even higher shift in the beam-beam phase for the proton beam. This large, nonlinear phase-shift will impair the quality of the proton beam due to the nonlinear resonance resulting from the large tune-spread.

In summary, multiple goals must be met to achieve the optics design of the electron beam. The machine requires high luminosity, a successful energy-recovery process demands a low electron beam emittance after collision, and the pinch effect needs to be small to assure the stability of the opposing proton beam. As we discussed, our findings demonstrate that for same electron beam waist size, a higher 
waist beta-function leads to larger luminosity, but worsens the final emittance by imposing a larger pinch effect, and consequently, a short lifetime for the proton beam. We need to compromise to reach our goals by choosing the proper optics functions.

Figures 9 and 10 show some of the important outcomes of optimizing the parameters. Each point in these figures corresponds to one initial electron-beam parameter and optics; we vary three parameters, viz., the initial electron-emittance, the waist beta-function $\beta^{*}$, and its position $s^{*}$.

Table 3. The parameters ranges used at the optimization of the electron IR optics design and the luminosity in eRHIC

\begin{tabular}{|c|c|}
\hline $\begin{array}{c}\text { Initial Electron rms Emittance } \\
\left(\times 10^{-9} \mathrm{~m}-\mathrm{rad}\right)\end{array}$ & {$[1 ; 8]$} \\
\hline Electron waist beta function $\beta^{*}(\mathrm{~m})$ & {$[0.1 ; 2]$} \\
\hline Electron Waist position $s^{*}(\mathrm{~m})$ & {$[-0.5 ; 0.5]$} \\
\hline
\end{tabular}

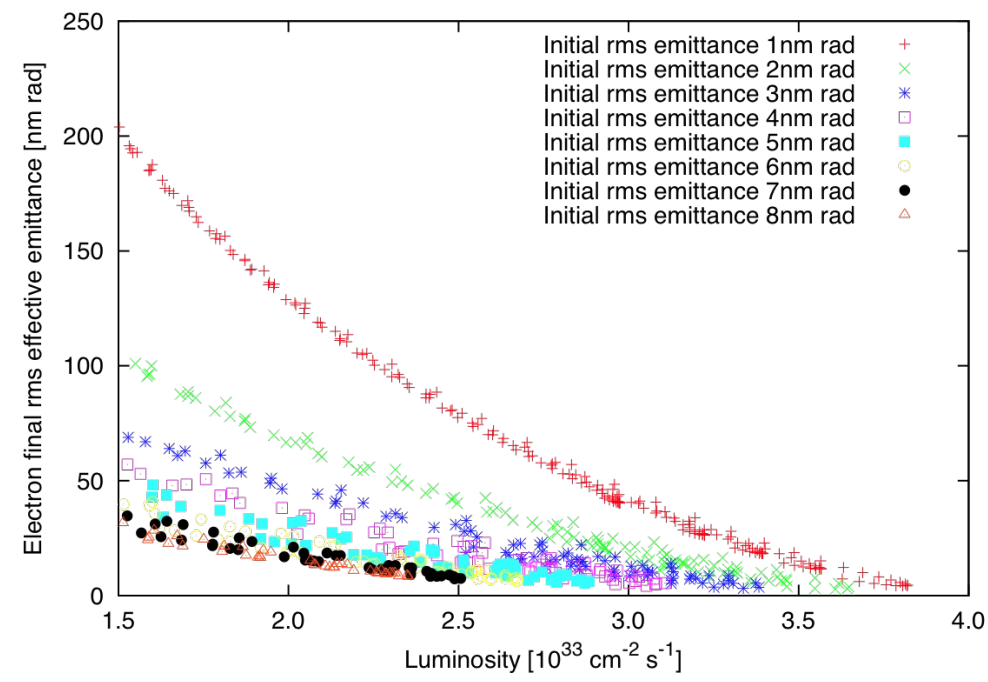

Figure 9. Electron beam rms effective emittance after collision plotted as a function of the resulting luminosity. 


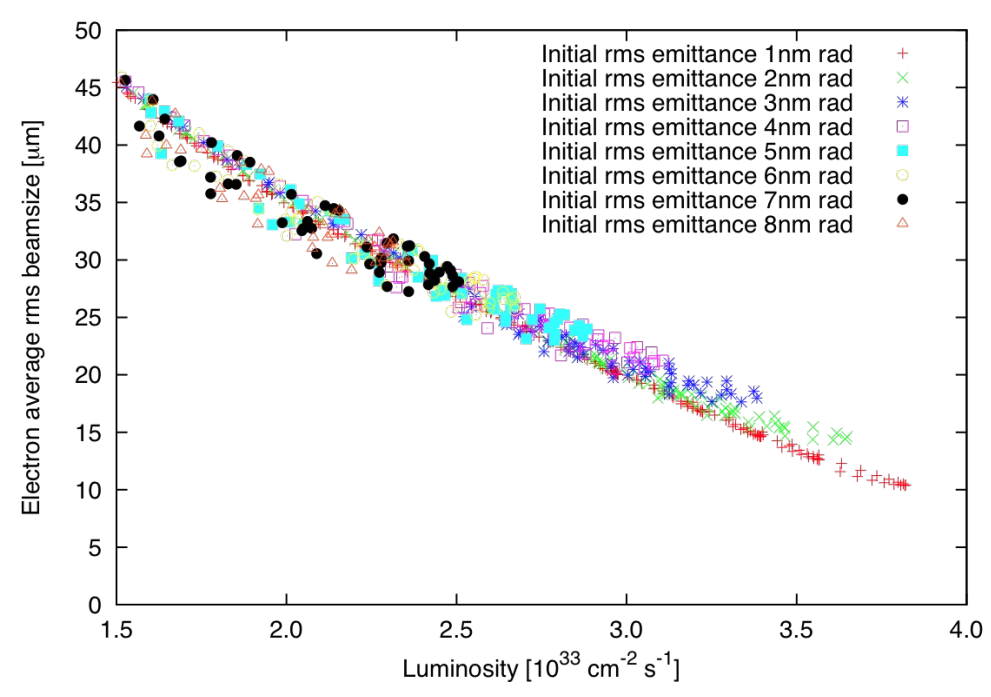

Figure 10. Plots of the rms electron-beam size during collision as a function of the resulting luminosity.

In figure 9, the final rms effective emittance is plotted as function of the resulting luminosity after considering the pinch and the hourglass effects. An interesting feature is that, for a fixed luminosity, a larger initial rms emittance leads to the lower final emittance. Further, at any given initial rms emittance, if the designed optics produces high luminosity, then, simultaneously, a smaller final rms emittance is obtained. Luminosity and the final rms emittance have a very simple relationship.

However, the pinch effect limits the realizable luminosity. Figure 10 plots the correlation between luminosity and the average size of the electron beam during the collision. A small electron beam enhances the beam-beam force acting on the protons, and causes a slow deterioration of the proton beam emittance. Simulations demonstrate that the limit of the average electron beam size is about 20 microns, corresponding to about $3 \times 10^{33} \mathrm{~cm}^{-2} \mathrm{~s}^{-1}$ luminosity. At smaller beam size the deterioration of the proton beam emittance becomes not acceptable.

On the basis of the results obtained from scanning of the electron beam parameters, we concluded that the initial emittance of $5 \mathrm{~nm}$ rad and $\beta^{*}=0.2 \mathrm{~m}$ present the optimal choice, which assure both an acceptable electron beam quality after collision and large luminosity. The shift of the electron beta-waist location $s^{*}$ from the center, towards the electron beam, produces somewhat large luminosity, as shown in figure 11. Original eRHIC IR design, based on $\beta^{*}=1 \mathrm{~m}$, was modified to satisfy the results of this studies, which led to the beam parameter set presented in Table 1. Table 1 


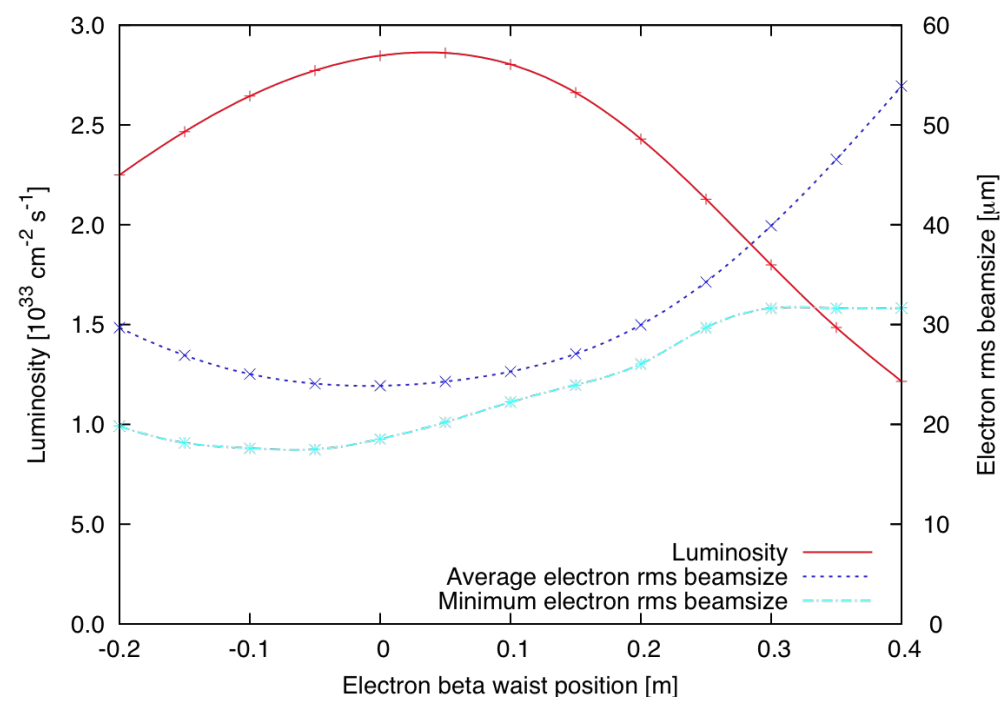

Figure 11. Dependence of luminosity and the electron beam size as function of the waist position of the beta function.

\subsubsection{Kink Instability}

Kink instability is a head-tail type instability [6,7] that arise from the beam-beam interaction between the two colliding beam. Through the interaction, the imperfection of the hadron beam can pass from the head of the beam to the tail and build up exponentially in the ring and form instability. Usually the dipole moment of the imperfection has the most important effect for the proton beam, since it has the lowest threshold.

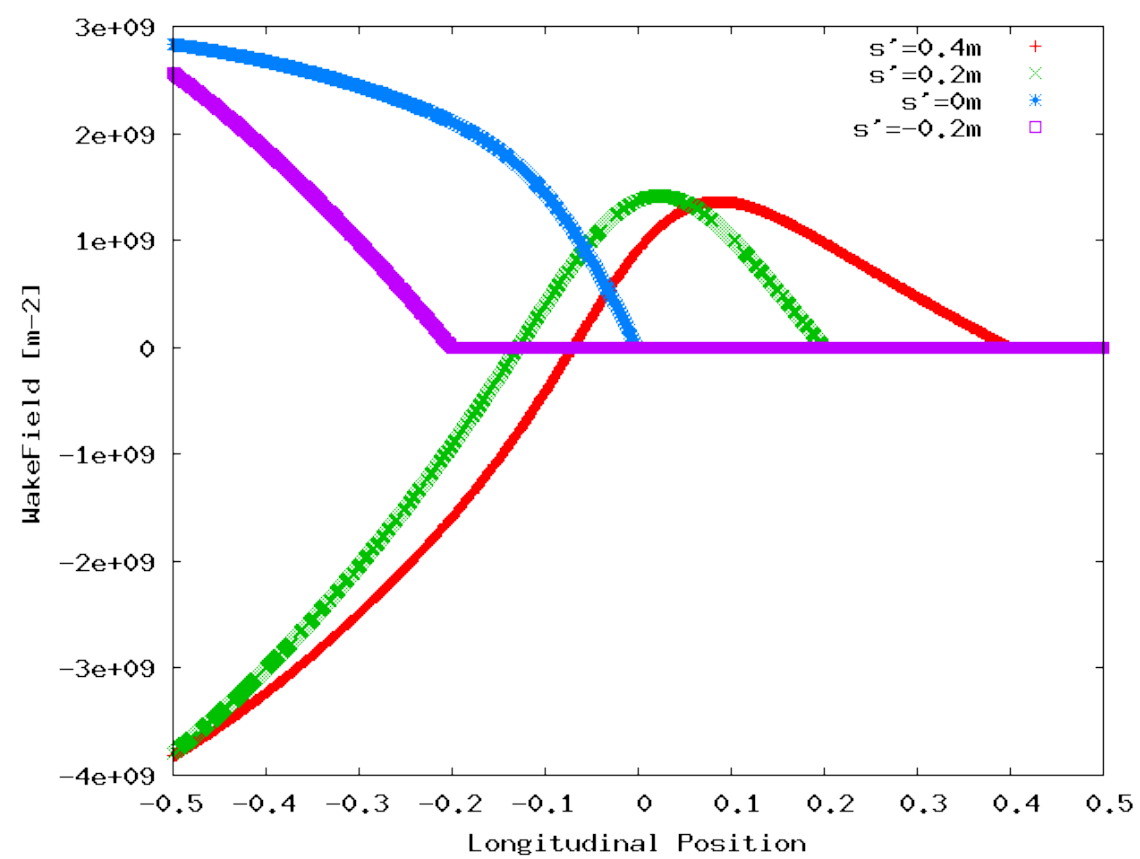

Figure 12. The wake field of a longitudinal Gaussian distribution.

Effectively, the interaction with the electron beam can be deemed as an 
interaction with a complicate wake field. This wake field depends on the proton beam distribution. For a uniform distribution with length $2 L$, the wake field has a simple sinusoidal form $W(s-\sigma)=\alpha \vee \kappa\left(\sigma-\sigma^{\prime}\right) H(\sigma-\sigma)$, where $k=\left(2 \phi_{\varepsilon} \Lambda\right)^{-1 / 2}$ is the focusing strength of the electron beam, $H$ is the Heaviside step function and $s$, presents the longitudinal position of the proton. In this special case, the wake field only depends on the longitudinal distance between heading and trailing particles. However, this does not hold for arbitrary proton longitudinal distribution. For a general distribution, the wake field is a function of both longitudinal positions instead of their difference. It can be derived from the simulation of the electron disruption study. Figure 12 reflects the wake field of the proton beam with a longitudinal Gaussian distribution. It has different shapes for various longitudinal position $s$ '.

The threshold of the instability needs to be determined from the wake field. The simplest estimation can be done theoretically. By using a constant wake field and 2 macro-particles to represent the proton beam, the threshold of the kink instability is given approximately by $D_{e} \xi_{\pi}<v_{\sigma}$ [8], where the disruption parameter of the electron beam, the beam-beam parameter of the proton beam and the proton synchrotron tune are involved. Although it is not a precise one, the estimation does reflect the basic feature of the head-tail instability. When no synchrotron motion is present, the system is always unstable. The faster the synchrotron motion is, the larger beam-beam strength is allowed.

For the real situation, simulation results can anticipate the threshold more precisely than the over-simplified analytical model. The simulation considers the real proton transverse and longitudinal distribution as well as a short electron bunch. Therefore the correct wake field and the nonlinearity of the interaction are taken into account. 


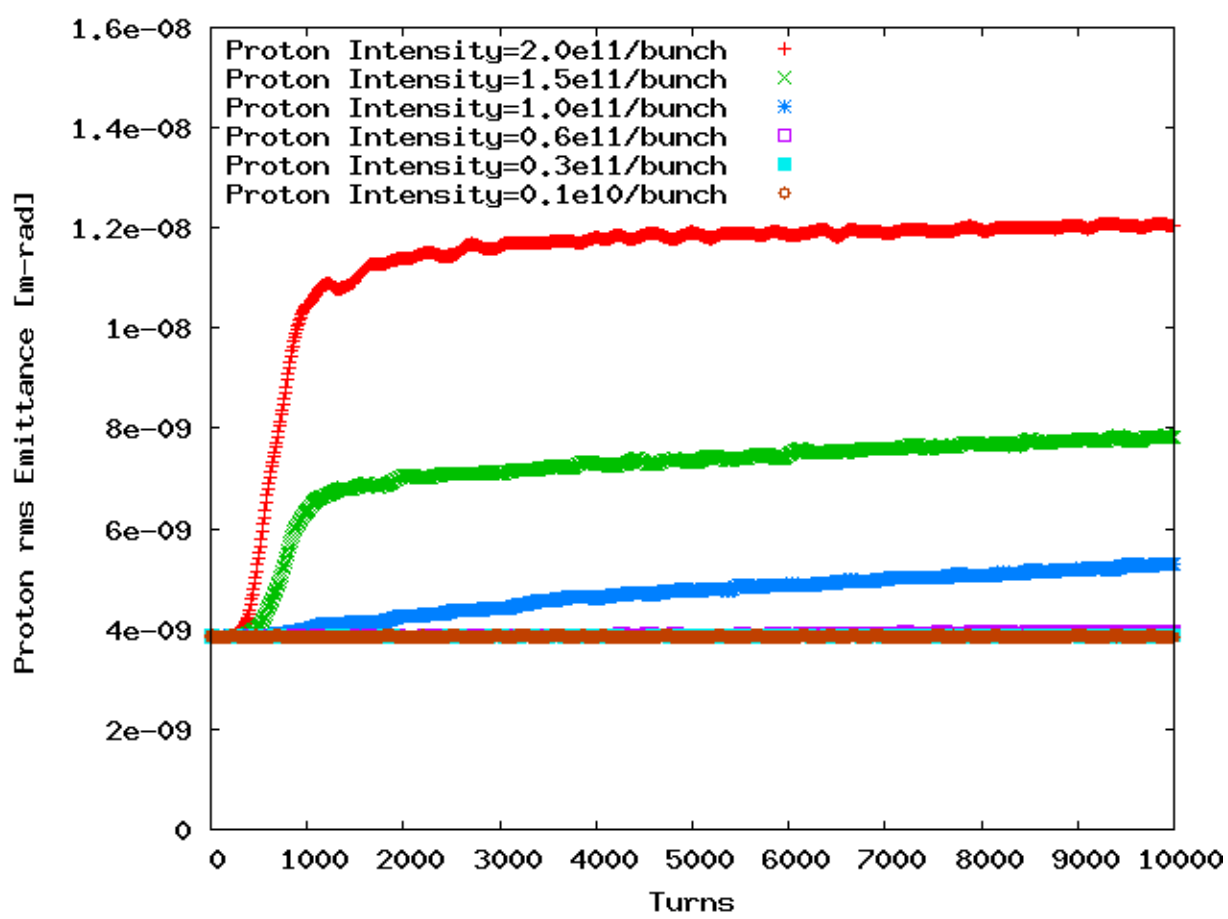

Figure 13. The proton rms emittance growth at different proton beam intensities.

Figure 13 shows the proton rms emittance growth under nonlinear beam-beam force with zero chromaticity for different proton beam intensities. Here the electron and proton parameters follow the nominal design values (Table 1) except proton beam intensity. The nominal proton beam intensity is $2.0 \times 10^{11}$ per bunch, which gives fastest emittance growth. The growth rate decreases as the proton intensity drops down. At very low intensity $\left(<3.0 \times 10^{10}\right.$ protons per bunch), no emittance growth is observed within calculation time. After rising quickly, the emittance growth in the unstable high intensity cases is suppressed by nonlinearity of the force.

Apparently, the eRHIC parameters exceed the threshold of the kink instability. To stabilize the beam, we can introduce tune spread to increase Landau damping. In reality, many effects, such as the nonlinear field in the lattice magnet elements, space charge effects and energy spread with a non-zero chromaticity, can bring up tune spread. In simulation, one of the easiest ways to generate controllable tune spread is the chromaticity. With a proper chromaticity of correct sign, the emittance growth can be suppressed and the proton beam becomes stable. 


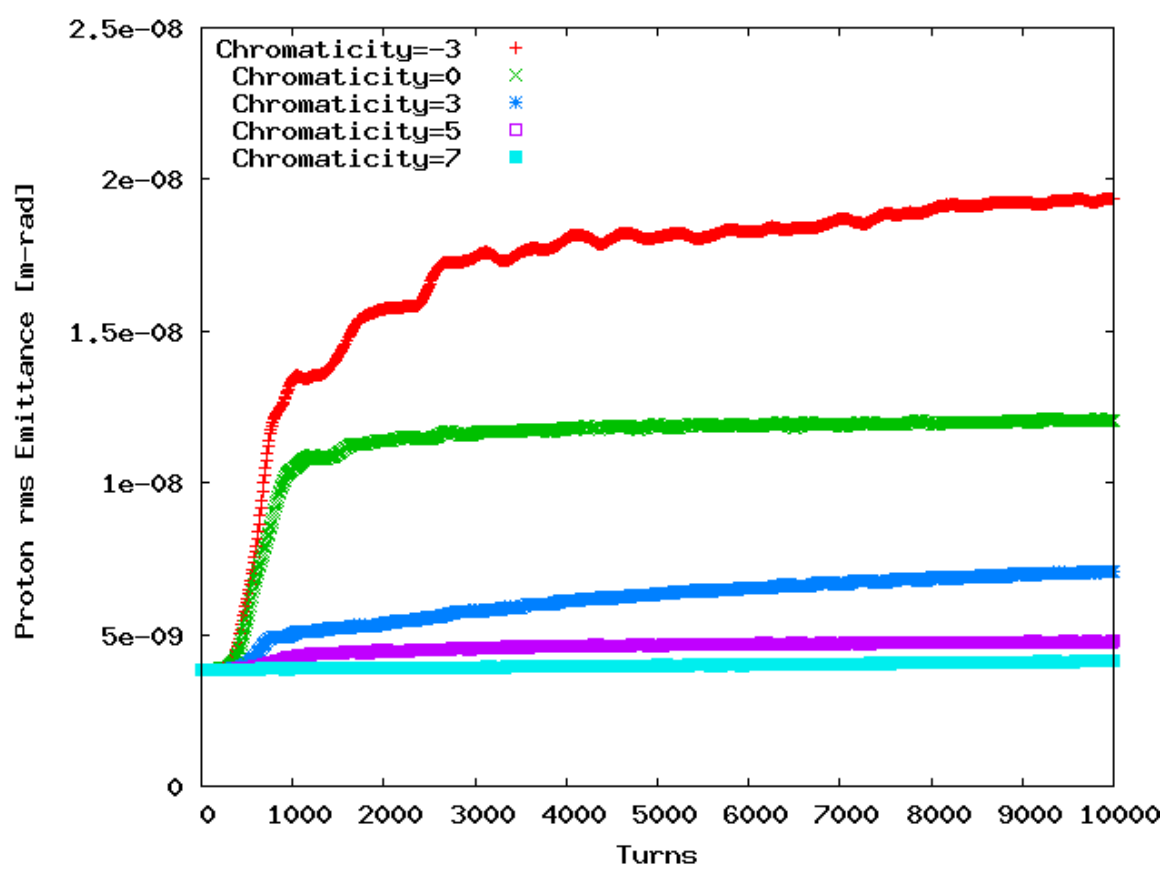

Figure 14. Proton rms emittance growth at different chromaticities with rms energy spread $5 \times 10^{-4}$ and bunch intensity $2 \times 10^{11}$.

Figure 14 shows the effect of the Landau damping, produced by the chromaticity induced tune spread. A negative chromaticity enhances the instability, as we expect for common head-tail instability, while a positive chromaticity does reduce the proton beam emittance growth. The figure indicates that the positive chromaticity of 5-7 can stabilize the proton beam in eRHIC.

In hadron machine operation, the large chromaticity may be unpleasant since the momentum aperture is limited. Even if the large tune spread can be produced by other means, it is always a concern that the nonlinear resonance can slowly deteriorate the beam. To avoid this problem, we consider a feedback system to eliminate the kink instability.

Figure 15 illustrates how the feedback system works. After each collision the orbit offset of the electron beam is measured by a Beam Position Monitor (BPM) and the value of the offset is transferred back to a kicker located before the interaction point. The next bunch of the electron beam, that collide with the same proton bunch, is kicked by amount proportional to the measured offset value. The factor, which defines the proportionality, is defined by the strength of the beam-beam interactions, by the location of the BPM and the kicker and by one turn transformation of the proton beam. 


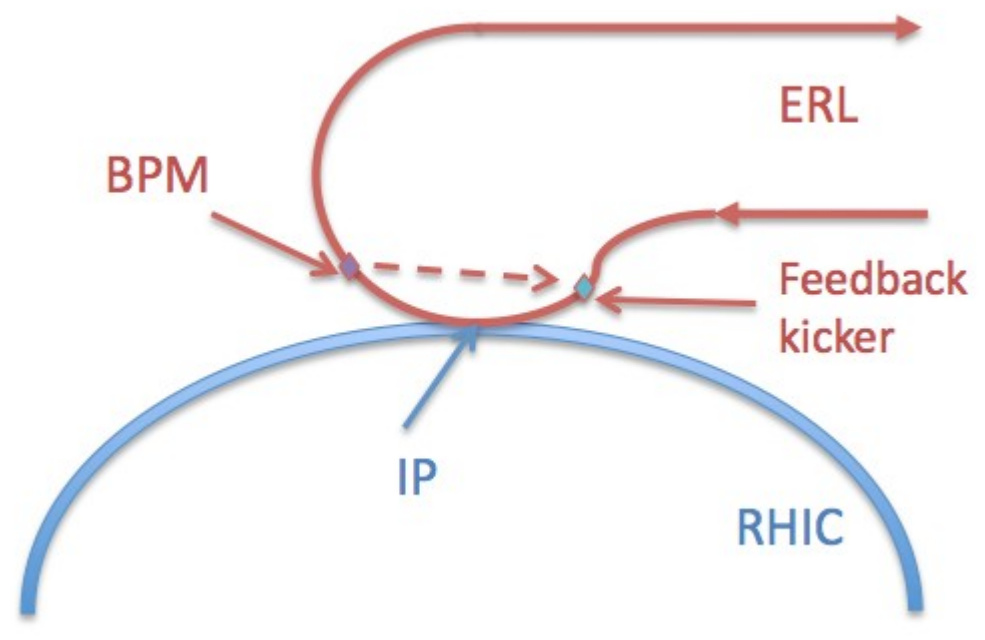

Figure 15. The scheme of the feedback system for mitigating the kink instability.

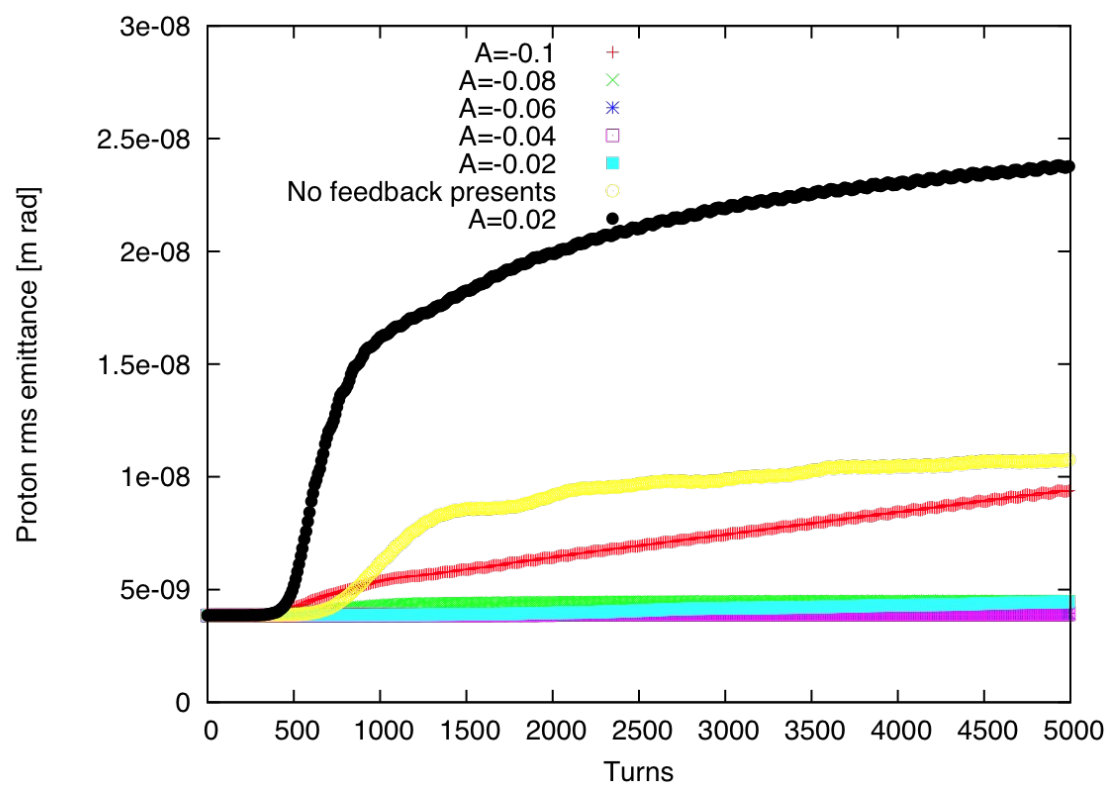

Figure 16. The simulation the kink instability with the state-of-art feedback system

Figure 20 shows the effect of the described feedback system to damp the kink instability. In the example, an electron bunch initial transverse offset, produced by the kicker, is determined by an offset measured at the BPM, amplified by a predefined factor A. No BPM and kicker errors are assumed to get these results. As expected, the factor $\mathrm{A}$ has to be a specific sign (negative in this example). An opposite sign will enhance the instability. The optimum case for stabilizing the beam is $A=-0.04$.

\subsubsection{Electron beam random errors}

In ERL-based eRHIC, a proton bunch meets millions electron bunches, which have slightly different parameters. The bunch parameter errors, characterizing the deviation of the bunch parameters from mean values, will affect the proton beam via 
beam-beam interaction $[9,10]$. Some errors, such as the electron beam intensity error, the rms beam size error affect the beam-beam parameter of the proton beam and can be called quadrupole errors. While other errors, such as the initial transverse position and momentum offsets can be called dipole errors because they lead to an extra kick to the proton beam.

By assuming a white noise spectrum of the electron bunch parameter errors, we can get simple estimation of the random effect on the proton beam. The dipole errors create a random walk motion of protons and ultimately lead to the linear growth of the rms beam size square over time. The growth is proportional to the amplitude of the noise.

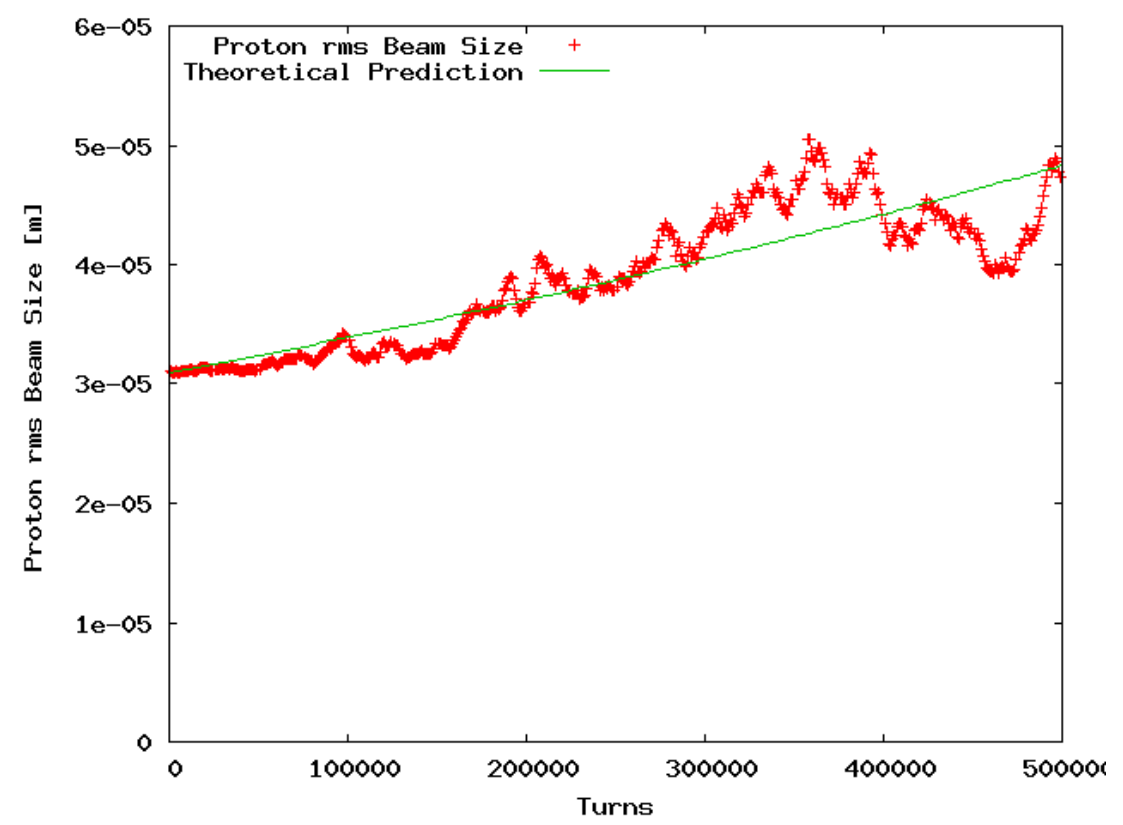

Figure 17. Proton rms beam size evolution at the presence of the electron bunch intensity noise and the comparison with the theoretical anticipation. Each beam size data is the average of 1000 turns.

The effect of quadrupole errors causes the exponential growth of the rms proton beam size. Figure 17 shows an example of simulation done with electron bunch intensity errors. The proton rms size increases exponentially and the result meets the theoretical anticipation. However, the real spectrum won't be a white noise. In general the noise contains more low frequency components. Once the actual frequency spectrum is determined, the detailed simulations will be done to evaluate the effect on the proton beam. The random error studies should provide tolerances on the fluctuations of electron beam parameters at the electron source and on the stability of elements in ERLs and transport lines. The evaluated tolerances and the element stability must be achieved to ensure a reasonable proton beam lifetime.

\section{References:}

[1] V.Ptitsyn, et al., "eRHIC Conceptual Design", Proceed. of Hadron Beam Workshop, Nashville, WGE01, p.388 (2008). 
[2] eRHIC Zeroth-Order Design Report, M. Farkhondeh and V. Ptitsyn, CA-D Note 142, 2004

[3] P. Grosse Wiesmann, Stanford Linear Accelerator Center Report No. SLAC PUB 4545, 1988

[4] S. A. Heifets, G. A. Krafft, and M. Fripp, Nuclear Instruments \& Methods in Physics Research Section a-Accelerators Spectrometers Detectors and Associated Equipment 295 (1990) 286.

[5] Y.Hao, V.Ptitsyn, "Effect of electron disruption in the energy recovery linac based electron ion collider" in Physical Review Special Topics: Accelerators and Beams 13, 071003 (2010).

Error: Reference source not found

[6] E. A. Perevedentsev and A. A. Valishev, Physical Review Special TopicsAccelerators and Beams 4 (2001)

[7] R. Li, et al., in Proceedings of the Particle Accelerator Conference, Chicago, (2001)

[8] Y. Hao, et al., in Proceedings of the International Particle Accelerator Conference, Kyoto, Japan (2010)

[9] Y. Hao, et al., in Proceedings of the Particle Accelerator Conference, Albuquerque, NM (2007)

[10] M. Blaskiewisz, BNL CAD/AP Notes, 363

Notice: This article has been authored by employees of Brookhaven ScienceAssociates, LLC under Contract No. DE-AC02-98CH10886 with the U.S. Departmentof Energy. The United States Government retains a non-exclusive, paid-up irrevocable,world-wide license to publish or reproduce the published form of this article, or allow others to do so, for the United States Government purposes. 